\title{
Cyclosporin A and sanglifehrin A enhance chemotherapeutic effect of cisplatin in $\mathrm{C} 6$ glioma cells
}

\author{
XUEZHE HAN ${ }^{1 *}$, SUNG HOON YOON ${ }^{2 *}$, YAN DING $^{2}$, TAE GYU CHOI ${ }^{2}$, WON JOON CHOI ${ }^{4}$, YUN HONG KIM $^{4}$, \\ YOUNG-JOO KIM ${ }^{5}$, YOUNG-BUHM HUH ${ }^{3}$, JOOHUN HA ${ }^{2}$ and SUNG SOO KIM ${ }^{2}$

\begin{abstract}
${ }^{1}$ Department of Neurosurgery, The First Hospital of Jilin University, Changchun 130-021, P.R. China; ${ }^{2}$ Department of Biochemistry and Molecular Biology (BK21 Project), Medical Research Center for Bioreaction to Reactive Oxygen Species and Biomedical Science Institute and ${ }^{3}$ Department of Anatomy, School of Medicine, Kyung Hee University, Seoul 130-701; ${ }^{4}$ Department of Anesthesiology and Pain Medicine, Kangbuk Samsung Hospital, School of Medicine, Sungkyunkwan University, Seoul 100-746; ${ }^{5}$ Department of Urology, College of Medicine,
\end{abstract} \\ Cheju National University, Cheju 690-756, Republic of Korea
}

Received September 16, 2009; Accepted November 16, 2009

DOI: $10.3892 /$ or_00000732

\begin{abstract}
Glioma is the most common type of brain tumors in adults, and treatment of high-grade gliomas is still palliative. Studies to date have revealed only modest effect in attenuating growth of these tumors with single agent therapy, but combination treatment appears to be more effective. Cyclophilin A (CypA), a target of immunosuppressive drugs cyclosporin A (CsA) and sanglifehrin A (SFA), is an intracellular protein that has peptidyl-prolyl cis-trans isomerase (PPIase) enzymatic activity. Previously, we showed that overexpressed CypA induced chemoresistance in cancer cells. Here we provide evidence that combination of cisplatin with either CsA or SFA synergistically enhances apoptotic cell death in C6 glioma cells, compared with single agent treatment. Enhanced apoptotic cell death is a result of an increase in ROS generation and a decrease in intracellular glutathione levels. Consistently, CypA knockdown by siRNA also enhances
\end{abstract}

Correspondence to: Dr Sung Soo Kim, Department of Biochemistry and Molecular Biology, School of Medicine, Kyung Hee University, no. 1, Hoegi-dong, Dongdae moon-gu, Seoul 130-701, Republic of Korea

E-mail: sgskim@khu.ac.kr

${ }^{*}$ Contributed equally

Abbreviations: CsA, cyclosporin A; GBM, glioblastoma multiforme; GFAP, glial fibrillary acidic protein; GSH, glutathione; GST, glutathione-S-transferase; HIF-1 $\alpha$, hypoxia-inducible transcription factor $1 \alpha$; HPLC, high performance liquid chromatography; NAC, $\mathrm{N}$-acetyl-L-cysteine; MDR1, multidrug resistance1; PARP, poly (ADP-Ribose) polymerase; PPIase, peptidyl-prolyl cis-trans isomerase; ROS, reactive oxygen species; SFA, sanglifehrin A

Key words: glioma, cylcosporin A, sanglifehrin A, cyclophilin A, peptidyl-prolyl cis-trans isomerase activity cisplatin-induced apoptosis. Immunohistochemical analysis showed increased expression of CypA in human glioblastoma multiforme, but not in normal human astrocytes. CypA was also shown to be up-regulated in C6 glioma cells during hypoxia. In conclusion, CsA or SFA in combination with cisplatin synergistically enhances cisplatin-induced apoptosis in C6 glioma cells via inhibition of PPIase activity of CypA, indicating that development of new drugs that selectively inhibit the CypA PPIase activity without immune suppression may facilitate alleviation of chemoresistance in treatment of high-grade glioma.

\section{Introduction}

Glioma is the most common type of brain tumors in adults, and accounts for $25 \%$ of all brain tumors $(1,2)$. Despite significant advances in cancer therapy, treatment of high-grade gliomas is still palliative. To date, the median survival for patients with high-grade gliomas, including glioblastoma multiforme (GBM; World Health Organization grade IV), is less than 1 year $(3,4)$. Therefore, improved systemic treatment strategies are urgently required.

Cyclophilin A (CypA), the prototypical member of the cyclophilin family, is a highly conserved protein in mammalian cells (5). CypA possesses enzymatic peptidyl-prolyl cis-trans isomerase (PPIase) activity, which is essential for protein folding in vivo. Although little is known about the function of CypA in cancer cells, it was recently reported that CypA is overexpressed in many cancer cells (6-11). In addition, our studies, as well as others, previously showed that overexpressed CypA protects cancer cells against cellular stresses, including hypoxia and cisplatin treatment, at least in part as a result of its antioxidant function (12-15). These reports show that CypA might be important for tumorigenesis in solid tumors. CypA is also an immunophilin and a cytosolic receptor for the immunosuppressive drugs cyclosporin A (CsA) (5) and sanglifehrin A (SFA) (16). CsA binds to CypA, and this complex inhibits calcineurin, a calcium-dependent phosphatase, that regulates the expression of various cytokine genes 
in activated $\mathrm{T}$ cells (17). The effects of CsA and SFA have been characterized in lymphocytes, but recent data suggest that CsA also affects signaling pathways in other cell types, such as adipocytes (18) and myocytes $(19,20)$. Recently, CsA and SFA have been reported to trigger tumor suppressor p53-mediated growth inhibition in some tumor cells (21), indicating their potential usefulness in cancer therapy.

Cisplatin is a DNA-damaging agent, and is among the most active and widely used cytotoxic anticancer drug (22). A major limitation in clinical use of cisplatin is acquisition of resistance by initially responsive tumors (23). Combinational use of cisplatin with other drugs, such as perillyl alcohol, other platinum(II) compounds, or AG1478, accelerates cisplatininduced apoptosis in several glioma cell lines (24-28). Moreover, recent studies have indicated that CsA enhances docetaxel-induced apoptosis in human gastric carcinoma cells (29). In this study, we demonstrate that either CsA or SFA significantly improves the anticancer effects of cisplatin by enhancing cisplatin-induced apoptosis in C6 glioma cells The molecular mechanism responsible for this enhanced apoptosis is closely related to inhibition of CypA's protective activity in cancer cells. We also show that CypA is overexpressed in GBM cells relative to normal astrocytes. Thus, the current study will facilitate opening new avenues of therapy in patients with high-grade gliomas.

\section{Materials and methods}

Materials. Dulbecco's modified Eagle's medium (DMEM), fetal bovine serum and 2'-7'-dichlorodihydrofluorescein diacetate (DCF-DA) were purchased from Invitrogen Life Technologies (Grand Island, NY), cisplatin, 3-(4,5-dimethylthiasol-2-yl)-2,5-diphenyltetrazolium bromide (MTT), Nacetyl-L-cysteine (NAC) and Hoechst 33342 were purchased from Sigma Aldrich (St. Louis, MO). Cyclosporin A (CsA) was purchased from Calbiochem (Darmstadt, Germany). Sanglifehrin A (SFA) was obtained from Novartis Pharma AG (Basel, Switzerland). Antibodies against poly (ADPribose) polymerase-1 (PARP), cytochrome $c$ and actin were purchased from Santa Cruz Biotechnology (Santa Cruz, CA). Antibody against caspase-3 was purchased from Assay Designs (Ann Arbor, MI). Antibodies against CypA and glial fibrillary acidic protein (GFAP) were from Abcam (Cambridge, MA). GenePORTER was purchased from Genlantis (San Diego, CA).

Cell culture. C6 rat glioma cells were grown in 10-cm diameter dishes containing DMEM supplemented with heat inactivated $10 \%(\mathrm{v} / \mathrm{v})$ fetal bovine serum, 100 units $/ \mathrm{ml}$ penicillin, and $100 \mu \mathrm{g} / \mathrm{ml}$ streptomycin. The cells were seeded in 6-well culture plates at a density of $1 \times 10^{4}$ cells $/ \mathrm{ml}$ for the microscopic assessment of cell death.

MTT assay. Cell viability was evaluated using the MTT conversion assay in a 12-well plate. The culture medium was replaced with $1 \mathrm{ml}$ medium containing $0.5 \mathrm{mg} / \mathrm{ml} \mathrm{MTT}$ and incubated for $60 \mathrm{~min}$ at $37^{\circ} \mathrm{C}$. The blue colored tetrazolium crystals resulting from mitochondrial enzymatic activity on MTT substrate were solubilized with $150 \mu 1 \mathrm{DMSO}$. The optical density was read at $595 \mathrm{~nm}$ in a microplate reader
(Bio-Rad, Richmond, CA). Cell survival was expressed as percentage of absorbance relative to that of untreated cells.

Hoechst 33342 staining. Cells were incubated for $30 \mathrm{~min}$ with Hoechst 33342 loading dye and fixed for $20 \mathrm{~min}$ in $4 \%$ formaldehyde. After washing with cold PBS three times, stained cells were monitored using the confocal laser microscope (Meta 510, Zeiss, Thornwood, NY). Apoptotic cells were identified by nuclear condensation and fragmentation.

Analysis of cellular ROS levels. ROS were measured using DCF-DA dye. The cells were grown to $80 \%$ confluence in complete medium, treated with or without cisplatin $(30 \mu \mathrm{M})$, loaded with $10 \mu \mathrm{M} \mathrm{DCF-DA}$ at $37^{\circ} \mathrm{C}$ for $30 \mathrm{~min}$ and then resuspended in $1 \mathrm{ml}$ of PBS. Fluorescence was measured by flow cytometer (Beckman Coulter Flow Cytometry). The mean DCF fluorescence intensity was measured with excitation at $488 \mathrm{~nm}$ and emission at $525 \mathrm{~nm}$. Untreated cells were used as a reference for ROS levels.

Glutathione determination. The cells were washed in PBS and harvested by scraping in 5\% MPA for the determination of intracellular glutathione (GSH) levels. After homogenization, the homogenates were incubated for $30 \mathrm{~min}$ on ice and centrifuged for $20 \mathrm{~min}$ at $18000 \mathrm{~g}$ to discard precipitated proteins. GSH content was assessed via HPLC (30). The levels of GSH were determined via comparison with standards and normalized to protein content.

Immunohistochemistry. Formalin-fixed, paraffin-embedded human tissues, including GBM and morphologically normal brain derived from surgical procedures and autopsy, respectively, were obtained from the Department of Neurosurgery at the First Hospital of Jilin University in China under approval by the Jilin University Institutional Review Board. Whole tissue sections (5- $\mu \mathrm{m}$ thick) were mounted on conventional charged glass slides. All sections were deparaffinized in three changes of xylene, and rehydrated through a descending series of ethanol. Heat antigen retrieval was done by immersing the section in $0.01 \mathrm{~mol} / 1$ sodium citrate $(\mathrm{pH}$ 6.0) in a pressure cooker (Nordic Ware, Minneapolis, MN) placed in a $1100-\mathrm{W}$ microwave set to 'high' for $15 \mathrm{~min}$. Following endogenous peroxidase blocking with a $0.03 \%$ hydrogen peroxide solution for $5 \mathrm{~min}$, $5 \%$ normal goat serum was applied for 30 min to block non-specific protein binding sites. The sections were then incubated for $1 \mathrm{~h}$ at room temperature with rabbit polyclonal anti-CypA or mouse polyclonal antiGFAP antibodies $(1: 1000)$. A biotin-free immunohistochemical staining detection technique was used for antibody visualization (EnVision+ System, HRP Rabbit kit, Dako, Carpinteria, CA). Subsequently, the sections were incubated for $30 \mathrm{~min}$ at room temperature with the goat anti-rabbit or horse anti-mouse secondary antibody conjugated with peroxidase-labeled polymers. The sections were visualized with application of 3,3-diaminobenzidine substrate chromagen solution. Pooled non-immune irrelevant rabbit or mouse IgG (Vector Laboratories, Burlingame, CA), applied at the same final concentration, was used as a negative control for all samples. Sections of morphologically normal human hepatic tissue were used as positive tissue controls for CypA expres- 
A

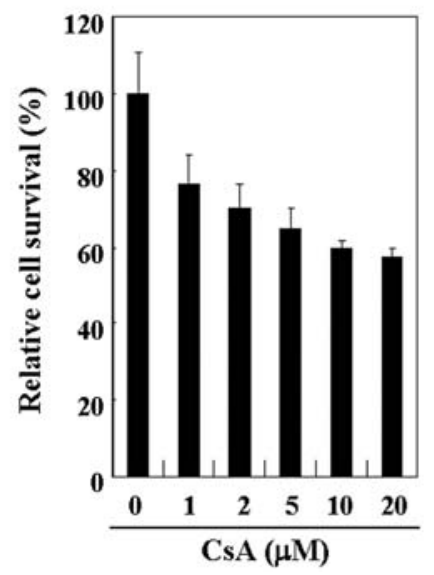

C

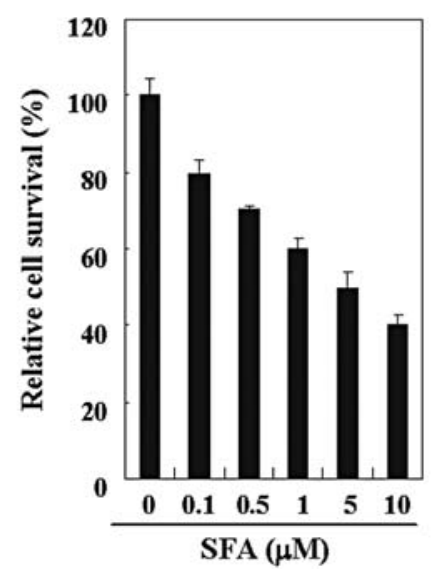

$\mathbf{E}$

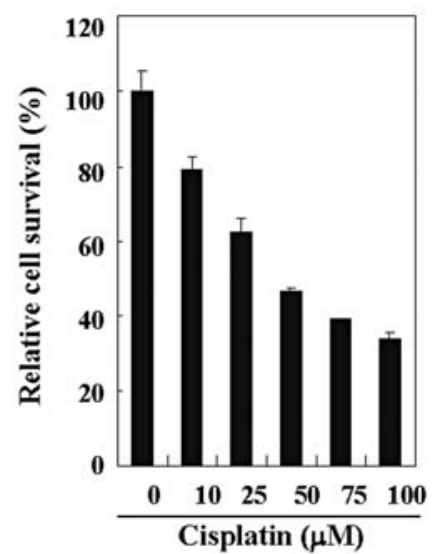

B

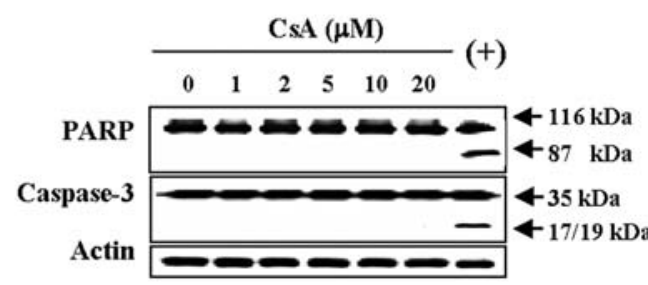

D

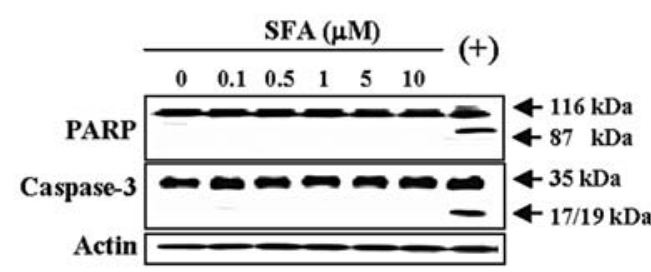

F

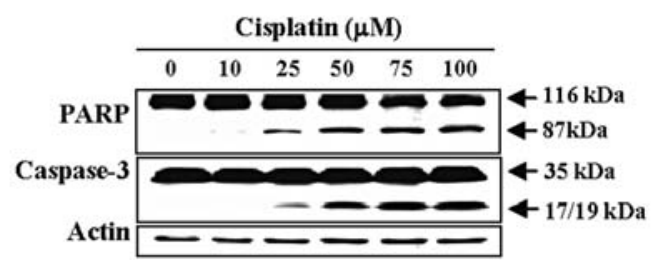

Figure 1. Dose responsiveness of C6 glioma cells. C6 cells were treated with the indicated concentrations of CsA (A), SFA (C) or cisplatin (E), and cell viability was determined via the MTT assay at $60 \mathrm{~h}$. Data represent the means \pm SE of at least three independent experiments. C6 cells were treated in the same ways, and then equal amounts of whole-cell extracts were resolved by SDS-PAGE and analyzed by Western blot analysis with antibodies specific to caspase-3 or PARP (B, D and F).

sion. Rabbit anti-vimentin antibody (Santa Cruz Biotechnology) was used as a positive assay control.

Peptidyl prolyl cis-trans isomerase assay. A modified method was adopted in order to determine PPIase activity in a coupled assay with chymotrypsin, as previously described (31). The peptide substrate $\mathrm{N}$-succinyl-Ala-Ala-Pro-Phe-p-nitroanilide was dissolved in trifluroethanol with $470 \mathrm{mM} \mathrm{LiCl}$ to create a 100-mM stock solution, which was then diluted to $4 \mathrm{mM}$ immediately prior to use. GST-CypA was diluted in $50 \mathrm{mM}$ HEPES and $86 \mathrm{mM} \mathrm{NaCl}, \mathrm{pH} 8.0$ (PPIase buffer), yielding a $2-\mu \mathrm{M}$ stock. In one cubic centimeter cuvette, $10 \mu \mathrm{l}$ of protein 
(20 nM final concentrations) was added to $860 \mu 1$ of PPIase buffer. The reaction was initiated via addition of $30 \mu 1$ $(120 \mu \mathrm{M})$ of peptide substrate followed by rapid mixing and the measurement of the absorption at $400 \mathrm{~nm}$ with an Amersham Biosciences spectrophotometer.

Preparation of total cellular fraction. Cells were washed three times with PBS and harvested in buffer (pH 7.4 $50 \mathrm{mM}$ Tris- $\mathrm{HCl}, 1 \%$ nonidet P-40, $0.25 \%$ Na-deoxycholate, $150 \mathrm{mM}$ $\mathrm{NaCl}, 1 \mathrm{mM}$ EDTA, $200 \mathrm{mM}$ PMSF, $200 \mathrm{mM} \mathrm{NaF}, 100 \mathrm{mM}$ $\mathrm{Na}_{3} \mathrm{VO}_{4}$ ). Cellular debris was removed via $15 \mathrm{~min}$ of centrifugation at $14000 \mathrm{~g}$. Whole cell extracts were snap-frozen in liquid nitrogen and stored at $-80^{\circ} \mathrm{C}$ until use for immunoblotting.

Small interfering RNA (siRNA). siRNAs specific to either CypA (CypA-siRNA) or control sequence (control-siRNA) were prepared by Eurogene tech (Intron Biotechnology, GyeongGi-do, Korea). siRNA $(0.5 \mu \mathrm{g})$ was transfected into cells using GenePorter transfection reagent. siRNA target sequences were as follows: CypA-siRNA (sense, 5'-UGACU UCACACGCCAUAAUdTdT-3'; antisense, 5'-AUUAUGGC GUGUGAAGUCAdTdT-3') and control-siRNA (universal negative control). The efficiency of siRNA-based interference of CypA was monitored by immunoblot.

Western blot analysis. Cell extracts were separated by SDSpolyacrylamide gel electrophoresis and transferred onto a nitrocellulose membrane. Transfer of proteins was assessed by Ponceau $\mathrm{S}$ red staining. After blocking, the membrane was incubated with the indicated primary antibody followed by incubation with a secondary antibody. Unless specified, actin protein was immunoblotted to standardize the amount of sample proteins for the Western blot analysis.

Statistical analysis. Results were expressed as means \pm standard errors (SE) from at least three independent experiments. Statistical analysis was performed by using Student's t-test. Unless otherwise indicated, $\mathrm{P}<0.05$ was deemed significant.

\section{Results}

Cyclosporin A, sanglifehrin A and cisplatin suppress C6 glioma cell proliferation. Although CsA and SFA were initially identified for their immunosuppressive activity, they were later shown to have direct antiproliferative effects on some tumor cells (32). Therefore, we wanted to determine if CsA or SFA could enhance cisplatin-induced apoptosis in C6 glioma cells. First, we observed the effects of CsA, SFA, and cisplatin on proliferation of C6 glioma cells using the MTT assay (Fig. 1A, C and E). As expected, CsA, SFA and cisplatin inhibited the proliferation of C6 glioma cells in a dosedependent manner.

Activation of caspases during apoptosis results in the cleavage of various cellular substrates including caspase-3 and PARP $(33,34)$. To determine if CsA, SFA or cisplatin induced apoptosis in C6 cells, we examined cleavage of caspase- 3 and PARP by Western blot analysis. The cleaved form of caspase- 3 and PARP proteins were undetectable in cells treated with CsA or SFA alone, even at relatively high concentrations $60 \mathrm{~h}$ after treatment (Fig. 1B and D). However, the cleaved forms of caspase- 3 and PARP proteins increased in a dose-dependent manner $60 \mathrm{~h}$ after cisplatin treatment, suggesting that cisplatin directly induces apoptosis in C6 glioma cells (Fig. 1F).

The combination of cisplatin and either cyclosporin A or sanglifehrin A induces the synergistic effects on growth inhibition and apoptosis of C6 glioma cells. We next investigated the effects of cisplatin in combination with either CsA or SFA on growth inhibition of C6 glioma cells. To examine the effect of single-agents in combination we used doses of $5 \mu \mathrm{M}$ CsA (Fig. 1A), $0.1 \mu \mathrm{M}$ SFA (Fig. 1C) and $10 \mu \mathrm{M}$ cisplatin (Fig. 1E), all of which produced only partial responses on their own.

Combining $10 \mu \mathrm{M}$ cisplatin with either $5 \mu \mathrm{M}$ CsA or $0.1 \mu \mathrm{M}$ SFA produced a synergistic effect on growth inhibition of C6 glioma cells as measured by the MTT assay (Fig. 2A). Since oxidative stress is responsible for the induction of apoptosis by cisplatin (35), the effect of the antioxidant NAC on cisplatin-induced growth inhibition was studied. Addition of $5 \mathrm{mM}$ NAC markedly rescued the combination-induced cell growth inhibition (Fig. 2A).

Apoptotic changes were analyzed via observation of cellular and nuclear morphological changes, as well as detection of PARP and caspase-3 cleavage. Cells treated with $10 \mu \mathrm{M}$ cisplatin and either $5 \mu \mathrm{M}$ CsA or $0.1 \mu \mathrm{M}$ SFA in combination were more rounded up, and exhibited more blebbing than those treated with cisplatin, CsA, or SFA alone (Fig. 2B). Nuclear staining with Hoechst 33342 demonstrated the typical features of apoptosis, including chromosomal condensation and nuclear fragmentation (Fig. 2C). Clearly, combining cisplatin with either CsA or SFA induced massive apoptosis, which was attenuated by pretreatment with NAC. As demonstrated above, CsA or SFA alone caused no cleavage of caspase- 3 or PARP. However, combination of cisplatin with either CsA or SFA led to increased cleavage of caspase- 3 and PARP, which is attenuated by NAC pretreatment (Fig. 2D), indicating that cisplatin-induced oxidative stress is required for combination-induced apoptosis.

Combined treatment of cisplatin with cyclosporin A or sanglifehrin A causes an increase in ROS generation and a decrease in intracellular glutathione levels. Using the fluorescent probe DCF-DA, we studied the effect of combining cisplatin with either CsA or SFA on ROS generation. Compared with drugs alone, the combination treatments caused a clear increase in intracellular ROS levels (Fig. 3A). This increased ROS was reversed by $5 \mathrm{mM}$ NAC pretreatment. Consistent with increased ROS, combinational treatment also reduced intracellular GSH levels (Fig. 3B) compared with cisplatin, CsA, or SFA alone, further supporting oxidative stress as the trigger of apoptotic cell death following combination treatment.

Cyclosporin A and sanglifehrin A sensitize cells to cisplatininduced apoptosis by suppressing PPIase activity of CypA. CsA (36) and SFA (37) have been shown to inhibit PPIase activity of cyclophilins. They also inhibit calcineurin activity 
B

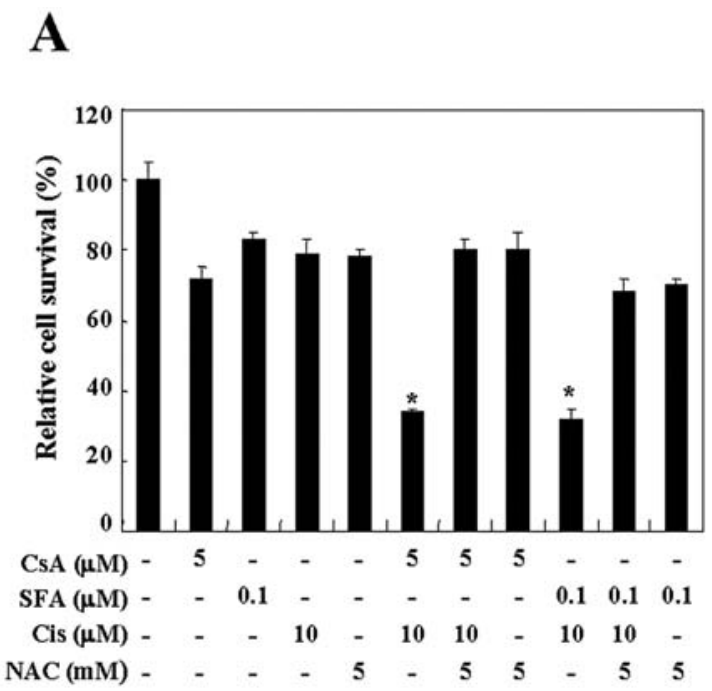

C

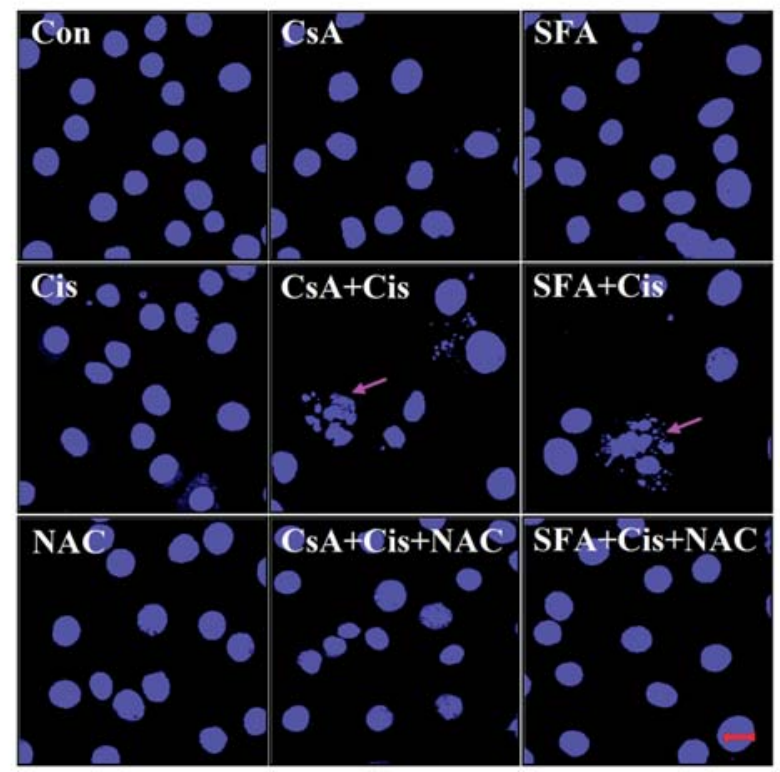

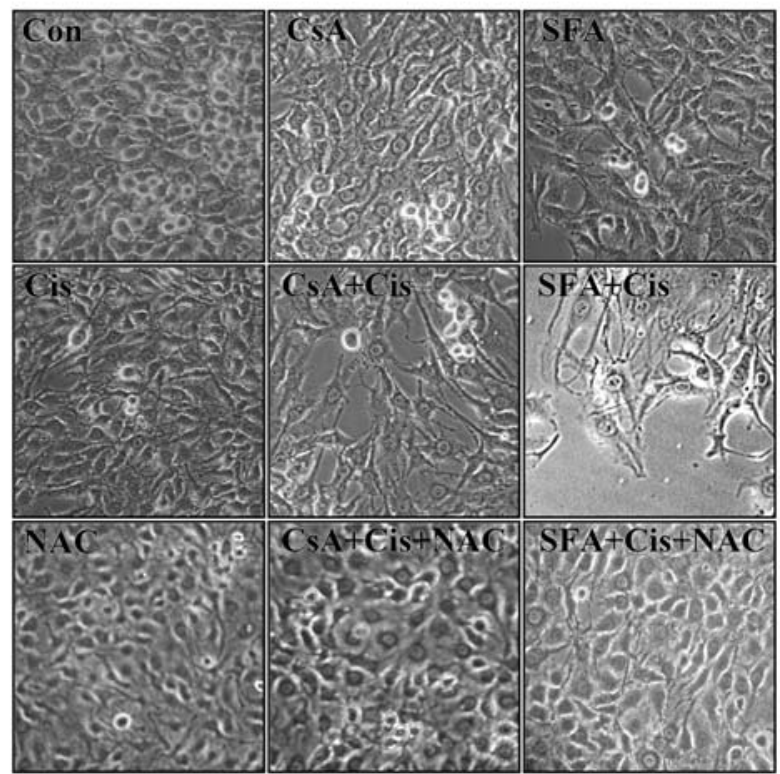

D

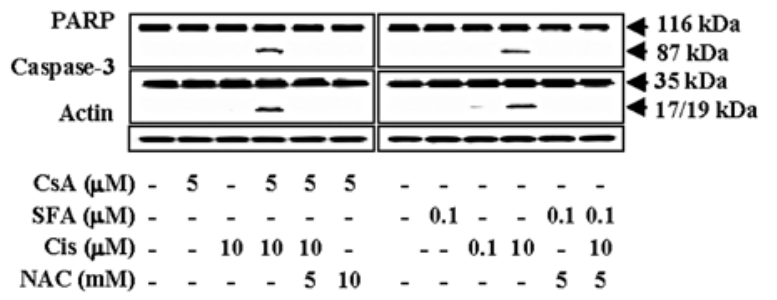

Figure 2. The combination of cisplatin with either cyclosporin A or sanglifehrin A induces the synergistic effects in C6 glioma cells. C6 cells were pre-

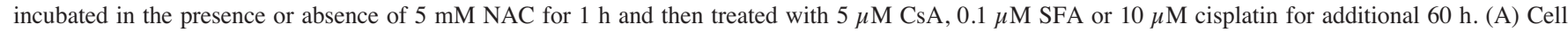
viability was assayed by MTT analysis. Data represent the means $\pm \mathrm{SE}$ of at least three independent experiments. ${ }^{*} \mathrm{P}<0.05$, versus cells treated with drugs alone. (B) Cell number was assessed by phase contrast microscopy. (C) After cells were stained with $10 \mu \mathrm{M}$ Hoechst 33342 for 15 min, nuclear staining images were captured by confocal microscopy. Arrow, apoptotic cells. Scale bars, $20 \mu \mathrm{m}$. (D) Western blot analysis with antibodies specific to caspase-3 or PARP.

after binding to CypA (17). NIM811, a CsA analogue, is also known to block the PPIase activity of CypA but not to be involved in the calcineurin pathway (14). To confirm the effect of CsA, SFA or NIM811 on inhibition of PPIase activity of CypA, we performed PPIase activity assays. As shown in Fig. 4, 20 nM GST-CypA fusion protein showed an increase in the rate of substrate isomerization, which was strongly inhibited by addition of $100 \mathrm{nM}$ CsA, 100 pM SFA or 100 nM NIM811, suggesting that each compound is capable of inhibiting the PPIase activity of CypA at quite low concentrations.
Next, to determine if CsA and SFA enhance cisplatininduced apoptosis by suppressing PPIase activity of CypA, we performed CypA knockdown experiments using CypA siRNA. The expression of CypA was almost $90 \%$ suppressed by specific siRNA interference (Fig. 5A). Knockdown of CypA and treatment with NIM811 showed results similar to those of CsA and SFA treatment in the MTT assay (Fig. 5B), suggesting that inhibition of PPIase activity of CypA is critical to the chemotherapeutic effects of CsA and SFA in combination with cisplatin. Hoechst 33342 staining (Fig. 5C) 
A

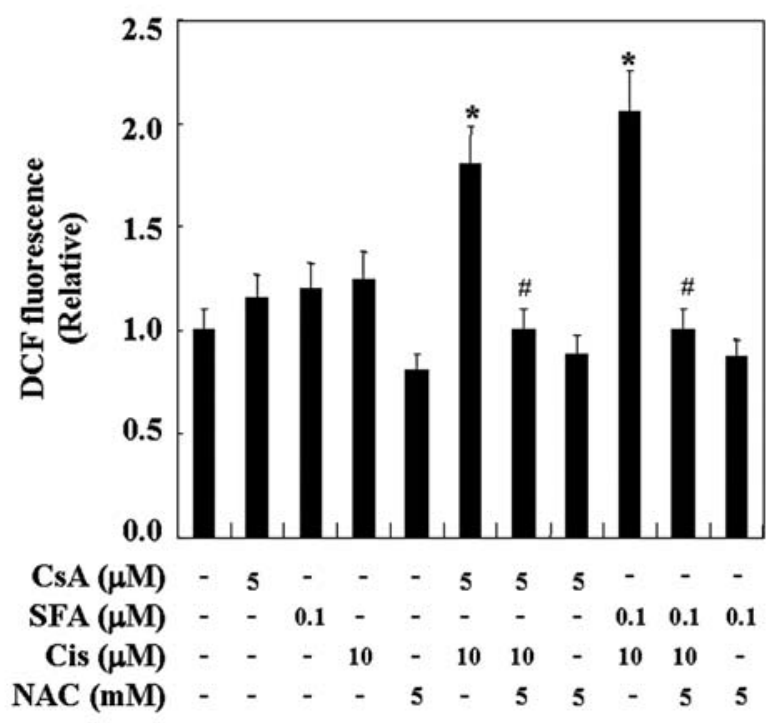

B

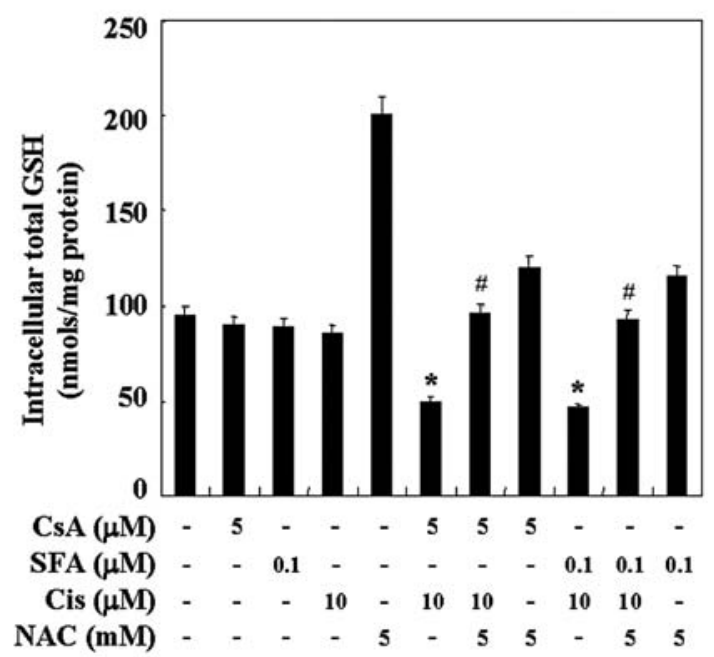

Figure 3. Combined treatment with cisplatin and either cyclosporin A or sanglifehrin A causes an increase in ROS generation and a decrease in intracellular glutathione levels. C6 cells were pre-incubated in the presence or absence of $5 \mathrm{mM}$ NAC for $1 \mathrm{~h}$ and then treated with CsA, SFA or cisplatin for additional $60 \mathrm{~h}$. Thereafter, the intracellular ROS levels (A) and total GSH levels (B) were assessed, as described in Materials and methods. Data represent the means $\pm \mathrm{SE}$ of at least three independent experiments. ${ }^{*} \mathrm{P}<0.05$, versus cells treated with drugs alone. ${ }^{~} \mathrm{P}<0.05$, versus cells treated with cisplatin and either CsA or SFA in combination.

and Western blot analyses of PARP and caspase-3 (Fig. 5D) in CypA siRNA transfected cells further confirmed this observation.

CypA expression in human glioblastoma multiforme and C6 glioma cells, but not in normal human astrocytes and upregulation of CypA expression during hypoxia in C6 glioma cells. Human samples including pathologically confirmed GBM and normal specimens were used to compare the expression of CypA by immunohistochemisty staining. To identify astrocytes, tissues were stained for GFAP, a specific marker for astrocytes. Analysis of normal human brain

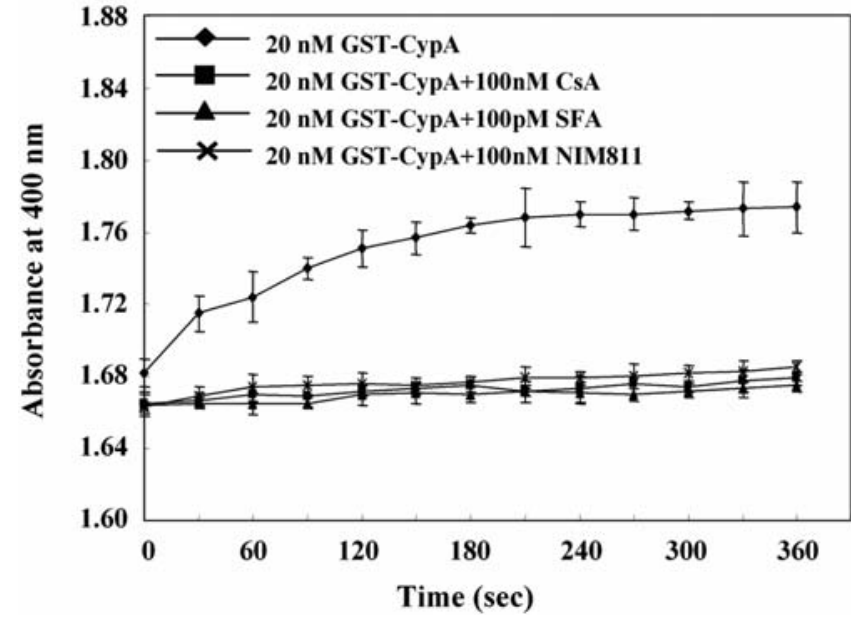

Figure 4. Inhibition of PPIase activity of cyclophilin A. Recombinant CypA at $20 \mathrm{nmol}$ was preincubated at $10^{\circ} \mathrm{C}$ for $5 \mathrm{~min}$ in $3.5 \mathrm{ml}$ of PPIase assay buffer with the concentrations of CsA, SFA or NIM811 as described in Materials and methods. The assay was started by the addition of peptide followed by rapid mixing and the measurement of the absorption at $400 \mathrm{~nm}$ with an Amersham Biosciences spectrophotometer.

sections revealed complete lack of CypA immunostaining within astrocyte cells. In contrast, strong immunoreactivity of CypA was observed in human GBM samples (Fig. 6A). Since one of the important pathological characteristics of GBM is increased hypoxia-induced necrosis (38), we next examined CypA protein expression in C6 glioma cells under normoxic or hypoxic conditions by Western blot analysis. There was a clear increase in CypA protein after cells were grown for $12 \mathrm{~h}$ under hypoxic conditions compared with normoxic conditions (Fig. 6B). Furthermore, we monitored the effect of CsA and cisplatin on the viability of C6 glioma cells under hypoxic conditions for $48 \mathrm{~h}$. As shown in Fig. 6C, the viability of C6 glioma cells was slightly decreased after $48 \mathrm{~h}$ of hypoxia exposure. Interestingly, pre-treatment with $5 \mu \mathrm{M}$ CsA before hypoxia exposure decreased cell survival significantly, while $10 \mu \mathrm{M}$ cisplatin did not show significant changes. Finally, combinational pre-treatment of cisplatin with CsA before hypoxia synergistically inhibited C6 glioma cell survival under hypoxic conditions. In contrast, all these treatments were less effective after cells were grown under hypoxia for $48 \mathrm{~h}$ (data not shown), confirming that upregulating CypA during hypoxia induces chemoresistance, as previously shown (15).

\section{Discussion}

Recent studies have proposed strategies incorporating the use of cisplatin in combination with other drugs such as perillyl alcohol, other platinum(II) compounds, and AG1478 to accelerate cisplatin-induced apoptosis in several glioma cell lines (24-28). Furthermore, combination of CsA with other anticancer drugs has been shown to enhance chemotherapeutic efficacy in leukemia and gastric carcinoma cells (29,39-41). In this study, we found that combining CsA or SFA with cisplatin synergistically enhances cisplatin-induced apoptosis in C6 glioma cells, suggesting that these combi- 


\section{A}

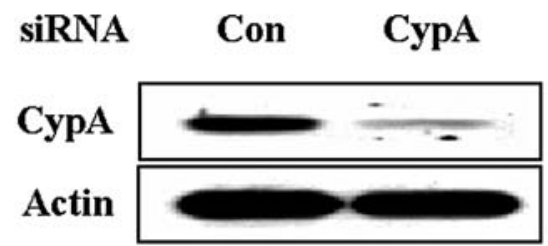

C

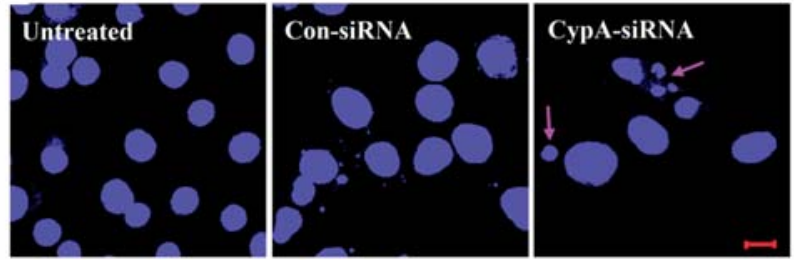

B

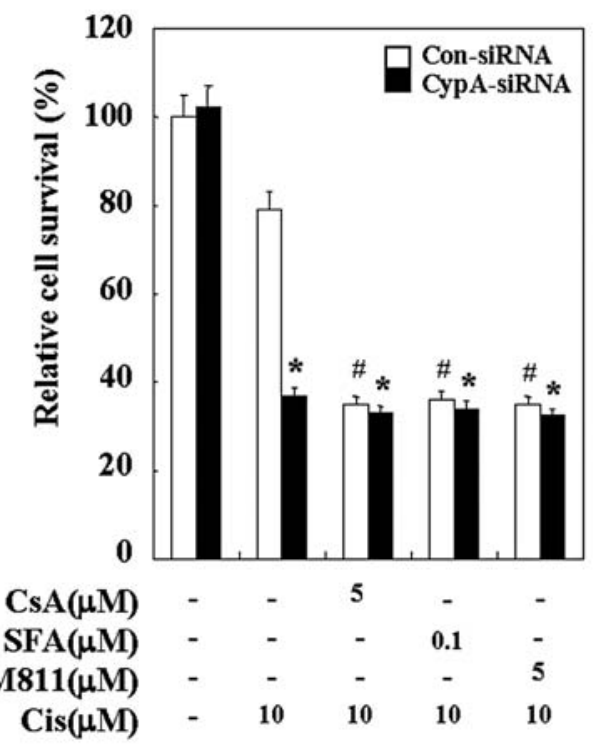

D

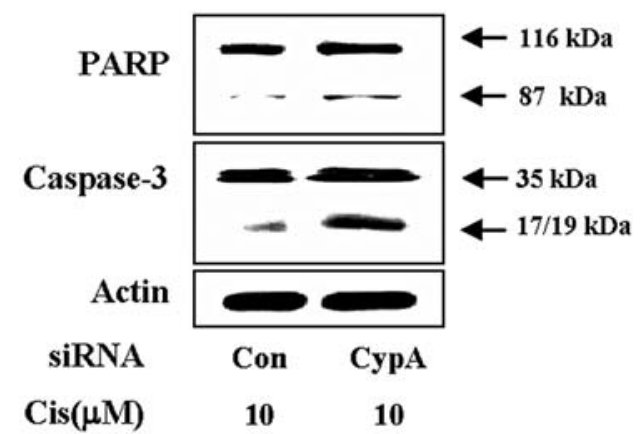

Figure 5. Effect of knockdown of cyclophilin A on cisplatin-induced apoptosis. (A) CypA expression level was monitored by immunoblotting in C6 glioma cells transfected with CypA-siRNA. (B) C6 cells transfected with siRNA specific to CypA were treated with $10 \mu \mathrm{M}$ cisplatin and either $5 \mu \mathrm{M}$ CsA or $0.1 \mu \mathrm{M}$ $\mathrm{SFA}$ in combination or alone for $60 \mathrm{~h}$. Cell viability was assayed by MTT. Data represent the means $\pm \mathrm{SE}$ of at least three independent experiments. "P $<0.05$, versus con-siRNA transfectants. ${ }^{*} \mathrm{P}<0.05$, versus CypA-siRNA transfectants. (C) After cells were stained with $10 \mu \mathrm{M}$ Hoechst 33342 for 15 min, nuclear staining images were captured by confocal microscopy. Arrows indicate apoptotic cells. Scale bars, $20 \mu \mathrm{m}$. (D) Western blot analysis with antibodies specific to caspase- 3 or PARP.

national therapies may enhance the therapeutic efficacy of cisplatin in GBM patients.

The molecular mechanisms of CsA chemotherapeutic effects have been delineated. Some groups report that CsA enhances the effect of anticancer drugs in leukemia cells by blocking the multidrug resistance pump (MDR1) (39-41). However, other groups suggest that CsA enhances taxotereinduced apoptosis mainly through an MDR1-independent pathway in GCTM-1 cells (29). Consistent with the latter group, we demonstrate here that CsA and SFA enhance cisplatin-induced apoptosis via inhibition of PPIase activity of CypA. Our conclusion is based on the following analyses. First, siRNA-based CypA knockdown aggravated cisplatininduced apoptosis. Second, combination of cisplatin with the CsA analogue NIM811, a selective inhibitor of CypA's PPIase activity without affecting calcineurin activity, also increased cisplatin-induced apoptosis. This finding indicates that the enhanced apoptosis is due to inhibition of the PPIase activity of CypA, but not due to inhibition of the calcineurin pathway, which is known to be immunosuppressive (42). Therefore, although we could not completely rule out the possibility that CsA suppresses cisplatin efflux in MDR-expressing cells, we concluded that the combination-induced synergistic effect is due to inhibition of the CypA PPIase activity. Since CypA possesses antioxidant activity (14), and the antioxidant NAC significantly abrogated the combination-induced apoptosis, it is likely that an increase in ROS levels and a decrease in GSH levels (Figs. 2-4) is a result of induced oxidative stress following inhibition of PPIase activity of CypA.

Astrocytes have been reported not to express CypA (43-45). Consistent with this report, we did not detect CypA expression in human normal astrocytes. However, for the first time we have demonstrated here that CypA is expressed in GBM and C6 glioma cells, which indicates that CypA expression is turned on during the process of transformation. Another factor in the increased expression of CypA in GBM may be hypoxia. We show here that CypA expression is up-regulated by hypoxia in C6 glioma cells, and have previously shown that CypA up-regulation is mediated by the HIF- $1 \alpha$ transcription factor in several cancer cells under hypoxic conditions 
A
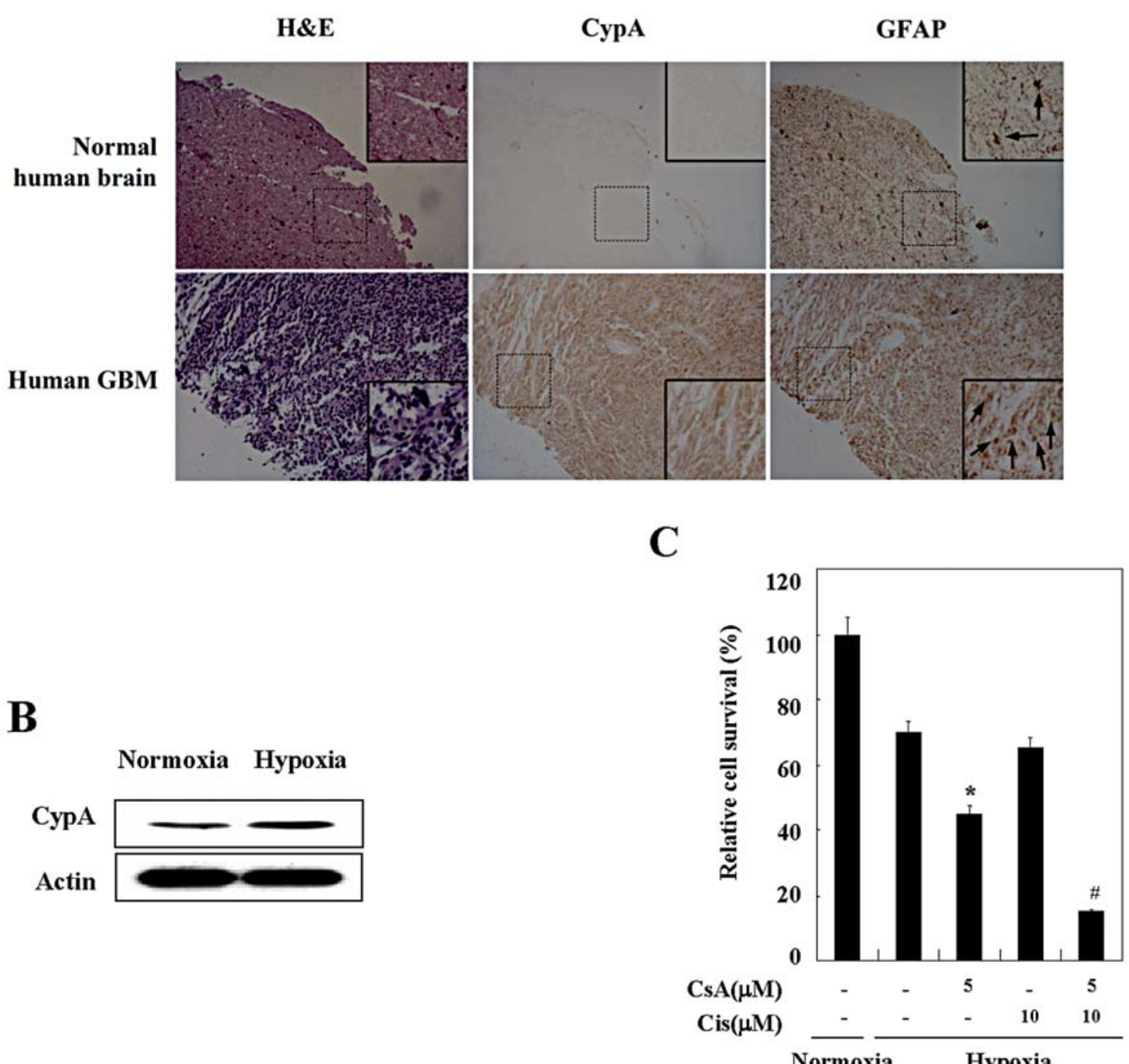

Normoxia Hypoxia

Figure 6. Cyclophilin A is detected in GBM tissues by immunohistochemistry and up-regulated by hypoxia. (A) GBM and normal human brain tissues were stained with either H\&E or antibody specific to CypA or GFAP (arrow). The normal glial tissues of the brain show no expression of CypA protein. In contrast, high levels of CypA protein expression are observed in GBM. Original magnifications, x100. Insets, magnifications, x400. (B) Total cell lysates $(10 \mu \mathrm{g})$ extracted after exposure to hypoxia for $12 \mathrm{~h}$ were subjected to Western blot analysis. (C) C6 glioma cells treated with CsA or cisplatin were exposed to hypoxia for $48 \mathrm{~h}$. Data represent means $\pm \mathrm{SE}$ of results from three independent experiments. ${ }^{*} \mathrm{P}<0.05$, versus cells exposed in hypoxia alone. $\mathrm{P}<0.05$, versus cells treated with CsA or cisplatin alone under hypoxic condition.

(15). In addition, one of the remarkable characteristics of GBM is the occurrence of hypoxia in the central region of tumors, which induces necrosis (38). These results led us to suggest that CypA may be epigenetically activated during transformation and further up-regulated by hypoxic conditions in GBM cells.

CsA shows a limited permeability through the intact blood-brain barrier, but a breakdown of this barrier has been observed in brain tumors. High-grade astrocytomas secrete the vascular endothelial growth factor, which increases angiogenesis, opening of tight junctions in highgrade astrocytoma microvessels, and endothelial cell permeability (46). The leaky blood-brain barrier in brain tumors may result in similar CsA concentrations in the brain as in blood. Therapeutic efficacy of CsA for experimental gliomas was apparent at clinically relevant concentrations. A daily CsA dose of 3-7 $\mathrm{mg} / \mathrm{kg}$ was used for many months without significant side effects in diverse patients including transplantation patients $(47,48)$ and patients with severe psoriasis $(49)$. Therefore, the strategy of combining cisplatin with either CsA or SFA at lower concentrations could be used for cancer patients in the clinic. One intriguing point to consider is that CsA and SFA may rather lessen survival rates of GBM patients due to their immunosuppressive effects. Therefore, we suggest new drugs that inhibit only PPIase activity of CypA without impairing immune activity need to be developed to stimulate cisplatininduced apoptosis. Although NIM811, a non-immunosuppressive CsA analogue, is currently in phase II clinical trials for the treatment of hepatitis $\mathrm{C}$, its effects on cancer cells still need to be largely investigated.

In conclusion, our data show that CsA or SFA synergistically enhances the chemotherapeutic effect of cisplatin in 
C6 glioma cells. Therefore, exploring the full potential of CypA's PPIase activity as a possible target for combination treatment with anticancer drugs could facilitate the alleviation of chemoresistance in high-grade glioma treatment. We are currently studying the synergistic effect of cisplatin combination therapy with CsA or SFA in a murine highgrade glioma model, and attempting to develop inhibitors of CypA's PPIase activity without immune intervention. Further understanding of this combination chemotherapy and development of more selective CypA's PPIase inhibitors will aid in better treatment outcomes for glioma patients.

\section{Acknowledgements}

This study was supported by the Korea Science and Engineering Foundation (grant no. R13-2002-020-02001-0 2007 to S.S.K.) and the Cheju National University in 2008 to Y.J.K.

\section{References}

1. Counsell CE and Grant R: Incidence studies of primary and secondary intracranial tumors: a systematic review of their methodology and results. J Neurooncol 37: 241-250, 1998.

2. Pobereskin LH and Chadduck JB: Incidence of brain tumours in two English counties: a population based study. J Neurol Neurosurg Psychiatry 69: 464-471, 2000.

3. Behin A, Hoang-Xuan K, Carpentier AF and Delattre JY: Primary brain tumours in adults. Lancet 361: 323-331, 2003.

4. Bredel M: Anticancer drug resistance in primary human brain tumors. Brain Res Brain Res Rev 35: 161-204, 2001.

5. Handschumacher RE, Harding MW, Rice J, Drugge RJ and Speicher DW: Cyclophilin: a specific cytosolic binding protein for cyclosporin A. Science 226: 544-547, 1984.

6. Li M, Wang H, Li F, Fisher WE, Chen C and Yao Q: Effect of cyclophilin A on gene expression in human pancreatic cancer cells. Am J Surg 190: 739-745, 2005.

7. Shen J, Person MD, Zhu J, Abbruzzese JL and Li D: Protein expression profiles in pancreatic adenocarcinoma compared with normal pancreatic tissue and tissue affected by pancreatitis as detected by two-dimensional gel electrophoresis and mass spectrometry. Cancer Res 64: 9018-9026, 2004.

8. Fillies T, Werkmeister R, van Diest PJ, et al: HIF1-alpha overexpression indicates a good prognosis in early stage squamous cell carcinomas of the oral floor. BMC Cancer 5 : 84,2004

9. Rey O, Baluda MA and Park NH: Differential gene expression in neoplastic and human papillomavirus-immortalized oral keratinocytes. Oncogene 18: 827-831, 1999.

10. Howard BA, Furumai R, Campa MJ, et al: Stable RNA interference-mediated suppression of cyclophilin A diminishes non-small cell lung tumor growth in vivo. Cancer Res 65 : 8853-8860, 2005.

11. Howard BA, Zheng Z, Campa MJ, et al: Translating biomarkers into clinical practice: prognostic implications of cyclophilin A and macrophage migratory inhibitory factor identified from protein expression profiles in non-small cell lung cancer. Lung Cancer 46: 313-323, 2004.

12. Seko Y, Fujimura T, Taka H, et al: Hypoxia followed by reoxygenation induces secretion of cyclophilin A from cultured rat cardiac myocytes. Biochem Biophys Res Commun 317: 162-168, 2004

13. Doyle V, Virji S and Crompton M: Evidence that cyclophilin-A protects cells against oxidative stress. Biochem J 341: 127-132, 1999.

14. Hong F, Lee J, Song JW, et al: Cyclosporin A blocks muscle differentiation by inducing oxidative stress and inhibiting the peptidyl-prolyl-cis-trans isomerase activity of cyclophilin A: cyclophilin A protects myoblasts from cyclosporin A-induced cytotoxicity. FASEB J 16: 1633-1635, 2002.

15. Choi KJ, Piao YJ, Lim MJ, et al: Overexpressed cyclophilin A in cancer cells renders resistance to hypoxia- and cisplatininduced cell death. Cancer Res 67: 3654-3662, 2007.
16. Fehr T, Kallen J, Oberer L, Sanglier JJ and Schilling W: Sanglifehrins A, B, C and D, novel cyclophilin-binding compounds isolated from Streptomyces sp. A92-308110. II. Structure elucidation, stereochemistry and physico-chemical properties. J Antibiot (Tokyo) 52: 474-479, 1999.

17. Ivery MT: Immunophilins: switched on protein binding domains? Med Res Rev 20: 452-484, 2000.

18. Ho IC, Kim JH, Rooney JW, Spiegelman BM and Glimcher LH: A potential role for the nuclear factor of activated $\mathrm{T}$ cells family of transcriptional regulatory proteins in adipogenesis. Proc Natl Acad Sci USA 95: 15537-15541, 1998

19. Musaro A, McCullagh KJ, Naya FJ, Olson EN and Rosenthal N: IGF-1 induces skeletal myocyte hypertrophy through calcineurin in association with GATA-2 and NF-ATc1. Nature 400: 581-585, 1999.

20. Semsarian C, Wu MJ, Ju YK, et al: Skeletal muscle hypertrophy is mediated by a $\mathrm{Ca}^{2+}$-dependent calcineurin signalling pathway. Nature 400: 576-581, 1999.

21. Pyrzynska B, Serrano M, Martinez A and Kaminska B: Tumor suppressor p53 mediates apoptotic cell death triggered by cyclosporin A. J Biol Chem 277: 14102-14108, 2002.

22. Jordan P and Carmo-Fonseca M: Molecular mechanisms involved in cisplatin cytotoxicity. Cell Mol Life Sci 57: 1229-1235, 2000.

23. Kartalou M and Essigmann JM: Mechanisms of resistance to cisplatin. Mutat Res 478: 23-43, 2001.

24. Rajesh D, Stenzel RA and Howard SP: Perillyl alcohol as a radio-/chemosensitizer in malignant glioma. J Biol Chem 278: 35968-35978, 2003.

25. Ross SA, Carr CA, Briet JW and Lowe G.: Transfer of 4'-chloro$2,2^{\prime}: 6^{\prime}, 2^{\prime \prime}$-terpyridine platinum(II) between human serum albumin, glutathione and other thiolate ligands. A possible selective natural transport mechanism for the delivery of platinum(II) drugs to tumour cells. Anticancer Drug Des 15: 431-439, 2000.

26. Lowe G, Droz AS, Vilaivan T, et al: Cytotoxicity of $\left(2,2^{\prime}: 6^{\prime}, 2^{\prime \prime}-\right.$ terpyridine)platinum(II) complexes to Leishmania donovani, Trypanosoma cruzi, and Trypanosoma brucei. J Med Chem 42: 999-1006, 1999.

27. Ahmadi R, Urig S, Hartmann M, et al: Antiglioma activity of $2,2^{\prime}: 6^{\prime}, 2^{\prime \prime}$-terpyridineplatinum(II) complexes in a rat modeleffects on cellular redox metabolism. Free Radic Biol Med 40: 763-778, 2006.

28. Huang PH, Mukasa A, Bonavia R, et al: Quantitative analysis of EGFRvIII cellular signaling networks reveals a combinatorial therapeutic strategy for glioblastoma. Proc Natl Acad Sci USA 104: 12867-12872, 2007

29. Nakahara C, Nakamura K, Yamanaka N, et al: Cyclosporin-A enhances docetaxel-induced apoptosis through inhibition of nuclear factor-kappaB activation in human gastric carcinoma cells. Clin Cancer Res 9: 5409-5416, 2003.

30. Ding Y, Choi KJ, Kim JH, et al: Endogenous hydrogen peroxide regulates glutathione redox via nuclear factor erythroid 2-related factor 2 downstream of phosphatidylinositol 3-kinase during muscle differentiation. Am J Pathol 172: 1529-1541, 2008.

31. Lee SM, Han X, Choi KJ, et al: A new method for purification of functional recombinant GST-cyclophilin A protein from E. coli. Indian J Biochem Biophys 45: 374-378, 2008.

32. Zhang LH, Youn HD and Liu JO: Inhibition of cell cycle progression by the novel cyclophilin ligand sanglifehrin A is mediated through the NFkappa B-dependent activation of p53. J Biol Chem 276: 43534-43540, 2001.

33. Orth K, Chinnaiyan AM, Garg M, Froelich CJ and Dixit VM: The CED-3/ICE-like protease Mch2 is activated during apoptosis and cleaves the death substrate lamin A. J Biol Chem 271: 16443-16446, 1996.

34. Cohen GM: Caspases: the executioners of apoptosis. Biochem J 326: 1-16, 1997.

35. Siddik ZH: Cisplatin: mode of cytotoxic action and molecular basis of resistance. Oncogene 22: 7265-7279, 2003.

36. Jeon SH, Piao YJ, Choi KJ, et al: Prednisolone suppresses cyclosporin A-induced apoptosis but not cell cycle arrest in MDCK cells. Arch Biochem Biophys 435: 382-392, 2005.

37. Clarke SJ, McStay GP and Halestrap AP: Sanglifehrin A acts as a potent inhibitor of the mitochondrial permeability transition and reperfusion injury of the heart by binding to cyclophilin-D at a different site from cyclosporin A. J Biol Chem 277: 34793-34799, 2002.

38. Lee CG, Heijn M, Di Tomaso E, et al: Anti-vascular endothelial growth factor treatment augments tumor radiation response under normoxic or hypoxic conditions. Cancer Res 60: 5565-5570, 2000. 
39. Bassan R, Lerede T, Borleri G, et al: Phase I trial with escalating doses of idarubicin and multidrug resistance reversal by shortcourse cyclosporin A, sequential high-dose cytosine arabinoside, and granulocyte colony-stimulating factor for adult patients with refractory acute leukemia. Haematologica 87: $257-263,2002$

40. Hirsch-Ernst KI, Ziemann C, Rustenbeck I and Kahl GF: Inhibitors of mdr1-dependent transport activity delay accumulation of the mdr1 substrate rhodamine 123 in primary rat hepatocyte cultures. Toxicology 167: 47-57, 2001.

41. Lum BL, Kaubisch S, Fisher GA, Brown BW and Sikic BI: Effect of high-dose cyclosporine on etoposide pharmacodynamics in a trial to reverse P-glycoprotein (MDR1 gene) mediated drug resistance. Cancer Chemother Pharmacol 45: 305-311, 2000.

42. Sigal NH, Dumont F, Durette P, et al: Is cyclophilin involved in the immunosuppressive and nephrotoxic mechanism of action of cyclosporin A? J Exp Med 173: 619-628, 1991.

43. Boulos S, Meloni BP, Arthur PG, et al: Evidence that intracellular cyclophilin A and cyclophilin A/CD147 receptormediated ERK1/2 signalling can protect neurons against in vitro oxidative and ischemic injury. Neurobiol Dis 25: 54-64, 2007.
44. Sameshima T, Nabeshima K, Toole BP, et al: Expression of emmprin (CD147), a cell surface inducer of matrix metalloproteinases, in normal human brain and gliomas. Int $\mathrm{J}$ Cancer 88: $21-27,2000$

45. Goldner FM and Patrick JW: Neuronal localization of the cyclophilin A protein in the adult rat brain. J Comp Neurol 372: 283-293, 1996.

46. Davies DC: Blood-brain barrier breakdown in septic encephalopathy and brain tumours. J Anat 200: 639-646, 2002.

47. Frei UA, Neumayer HH, Buchholz B, Niese D and Mueller EA: Randomized, double-blind, one-year study of the safety and tolerability of cyclosporine microemulsion compared with conventional cyclosporine in renal transplant patients. International Sandimmun Neoral Study Group. Transplantation 65: 1455-1460, 1998.

48. Levy G, Burra P, Cavallari A, et al: Improved clinical outcomes for liver transplant recipients using cyclosporin monitoring based on 2-hr post-dose levels (C2). Transplantation 73: 953-959, 2002.

49. Koo J: A randomized, double-blind study comparing the efficacy, safety and optimal dose of two formulations of cyclosporin, Neoral and Sandimmun, in patients with severe psoriasis. OLP302 Study Group. Br J Dermatol 139: 88-95, 1998. 